\title{
Improve efficiency of harvesting random energy by snap-through in a quad-stable harvester
}

\author{
Zhi-yong Zhou, Wei-yang Qin*, Pei Zhu \\ Department of Engineering Mechanics, Northwestern Polytechnical University, Xi'an 710129, \\ People's Republic of China
}

\begin{abstract}
In response to the defects of the bi-stable energy harvester $(\mathrm{BEH})$, we develop a novel quad-stable energy harvester $(\mathrm{QEH})$ to improve the harvesting efficiency. The device is made up of a bimorph cantilever beam having a tip magnet and three external fixed magnets. By introducing the repulsion forces between magnets, the function of potential energy of the QEH is given. It owns four potential wells. It is proved that the quad-stable harvester can cross the barriers and realize snap-through easier. Validation experiments were performed by frequency sweeping and random excitations. Results show that compared to the BEH the frequency bandwidth of snap-through of the novel device is much wider. The QEH can make a dense snap-through in response under random excitation, and give out a large output voltage. This shows that the proposed QEH is more effective in energy harvesting applications.
\end{abstract}

Keywords: quad-stable energy harvester; snap-through; piezoelectric beam; magnet

\section{Introduction}

Piezoelectric energy harvesting has become an active field of research in the application of environment control and monitoring [1-5]. For example, most of small autonomous electronic devices such as wireless sensor nodes have no access to an outside power source, hence the vibration based energy harvesters, which transforms ambient kinetic energy into electric power by using piezoelectric, electromagnetic and electrostatic mechanisms [6-10]. Conventional linear piezoelectric energy harvesting devices only work optimally near their resonance frequencies with a narrow frequency bandwidth around a particular resonant frequency. However, the vibration energy existing in environments generally has a wide band of frequency. So these narrowband devices are not fit for the practical application. Recently the bi-stable characteristics of some systems are utilized to harvest environmental vibration energy. The typical bi-stable energy harvester (as shown in Fig. 1), which has two stable equilibrium positions, has been studied widely. The snap-through phenomenon of it can provide a way to improve energy harvesting efficiency [11-13].

*Corresponding author. Tel.: +86 15229030828, +86 13186125796.

E-mail address: zzy569@163.com, qinweiyang@aliyun.com (W.Y. Qin). 


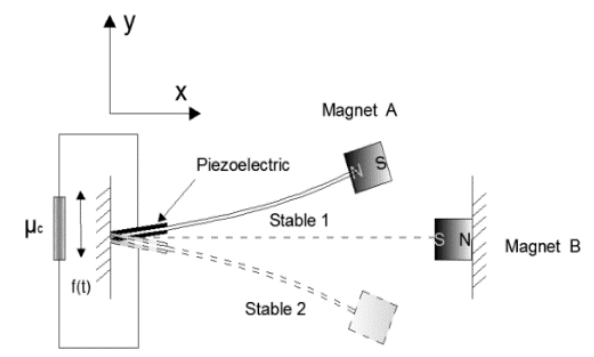

Fig. 1. Bi-stable cantilever piezoelectric energy harvester (BEH) with two stable states.

To realize the bi-stable characteristic, magnets are highly regarded for its simplicity and high performance. Magnetic forces have been frequently adopted to alter the stiffness of the energy harvester to enhance the conversion abilities. And magnets can be used to tune the resonant frequency of the energy harvester as well, which is ideally suited to efficiently harvest energy from ambient excitations with slowly varying frequencies $[14,15]$. Aline investigated the influence of nonlinearities in energy harvesting from a piezo-magnetoelastic structure subjected to random vibrations [16]. Erturk presented theoretical and experimental investigations on high-energy orbits in the piezo-magnetoelastic energy harvester over a range of excitation frequency $[17,18]$. Tang exploited a pair of magnets in repulsive and attractive configurations to enhance the vibration amplitude and the bandwidth of frequency [19].

To further enhance the performance of energy harvester, some studies focused on using adjustable or movable magnets. Lin investigated a magnetically coupled piezoelectric cantilever beam in which the displacement of the fixed magnet could be altered to achieve off-resonance to enhance the broadband frequency response [20]. Zhou et al. [21] investigate a magnetically coupled nonlinear piezoelectric energy harvester by altering the angular orientation of its external magnets for enhanced broadband frequency response. Tang and Yang introduced a magnetic coupled piezoelectric energy harvester in which the magnetic interaction is produced by a magnetic oscillator [22]. Gao conceived a kind of structure with an elastic support external magnet and proved that elastic support systems had better power output performance than rigid support systems when excited at low-intensity vibrations [23]. Jung investigated piezoelectric energy harvester with two rotatable external magnets. The results show that the angle of the external magnet and separation distance between the tip magnet and external magnets could alter the potential energy of harvester system [24]. It is shown that this design can broaden the harvesting bandwidth of frequency more effectively than the device with one fixed magnet. The device has the capability of snapping from one stable state to the other one at a low intensity excitation. Such snap-through motion can bring about a large-amplitude vibration and shows a good performance in energy harvesting.

But there exists a difficult point for the bi-stable harvester. With the increase of the distance between the two potential wells, the amplitude of snap-through will become large, but the barrier height increase as well. This hinders BEH in breaking through the constraint of potential barriers and thus it often undergoes an intrawell 
oscillation. Snap-through motion could improve the energy harvester performance [18]. So the harvester should be designed to make snap-through happen easily. The key parameters influencing the occurrence of snap-through are the depth and width of potential energy wells. The quad-stable system has four potential energy wells (two inner wells and two outer ones) in total. Snap-through motions crossing the nonadjacent potential wells (especially crossing over all four potential energy wells) can produce the relatively larger vibration amplitudes.It has been proven that the quad-stable device has the shallower and wider potential wells [25]. Therefore the quad-stable harvester having the low and wide potential wells may possess better capability of extracting electrical energy from ambient vibration than the BEH.

This paper proposes a novel quad-stable energy harvester $(\mathrm{QEH})$. The theoretical analyses and validation experiments are carried out. The results show that the QEH has a wide frequency bandwidth of large output voltage, even at a relatively low excitation. Compared with the BEH, the QEH could easily realize the snap-through motion between the potential wells and generate high output voltages.

\section{QEH model and potential energy analyses}

As shown in Fig. 2, the QEH device is composed of a bimorph cantilever beam with a tip magnet $\mathrm{D}$ and three fixed magnets $(\mathrm{A}, \mathrm{B}, \mathrm{C})$. By adjusting the positions of fixed magnets and the distance between the tip magnet and the fixed ones, the quad-stable equilibrium positions can emerge in the static state.

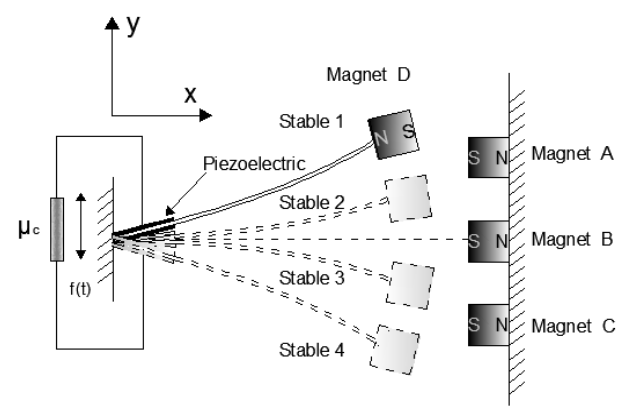

Fig. 2. Quad-stable cantilever piezoelectric energy harvester (QEH) with four stable states.

For the QEH device, the magnets can be modeled as point dipoles when calculating magnetic potential energy. The magnetic moment vector $\boldsymbol{m}$ is dependent on the magnet's volume $V$, and its magnetization $\boldsymbol{M}$ is related to the magnet's residual flux density $\boldsymbol{B}_{r}, \quad \boldsymbol{M}=\boldsymbol{B}_{r} / \mu_{0}$, where $\mu_{0}=4 \pi \times 10^{-7} \mathrm{Hm}^{-1}$ is the magnetic permeability constant [26-28]. Let $\boldsymbol{m}_{i}$ represent the magnetic moment vector of magnet $i$ (A, B or $\mathrm{C})$, then the potential energy of magnetic field generated by the three external magnets (A, B and C) upon magnet $\mathrm{D}$ can be given by

$$
U_{m}=U_{m A D}+U_{m B D}+U_{m C D}=-\boldsymbol{B}_{A D} \cdot \boldsymbol{m}_{D}-\boldsymbol{B}_{B D} \cdot \boldsymbol{m}_{D}-\boldsymbol{B}_{B D} \cdot \boldsymbol{m}_{D}
$$

where $\boldsymbol{B}_{i D}$ is the flux density generated by magnet $i$ upon magnet D. $\boldsymbol{B}_{i D}$ can be calculated by 


$$
\boldsymbol{B}_{i D}=-\frac{\mu_{0}}{4 \pi} \nabla \frac{\boldsymbol{m}_{i} \cdot \boldsymbol{r}_{i D}}{\left\|\boldsymbol{r}_{i D}\right\|_{2}^{3}}
$$

where $\boldsymbol{r}_{i D}$ is the vector directed from the magnetic moment source of $i$ to that of D. $\left\|\boldsymbol{r}_{i D}\right\|_{2}$ and $\nabla$ denote Euclidean norm and vector gradient operator respectively. So the potential energy created by magnet $i$ upon magnet $\mathrm{D}$ can be written as

$$
U_{m i D}=-\boldsymbol{B}_{i D} \cdot \boldsymbol{m}_{D}
$$

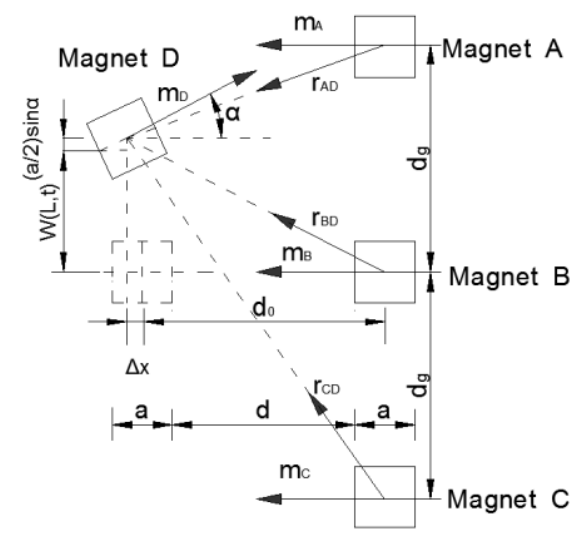

Fig. 3. Geometric configuration of the tip magnet and three external permanent magnets.

The geometric configuration of the tip magnet and three external permanent magnets is as shown in Fig. 3. We now need to give the horizontal and vertical displacements of magnet $\mathrm{D}$. Let $w(x, t)$ denote the transverse displacement of beam at position $x$ and instant $t$. Then the tip magnet $\mathrm{D}$ translates a distance $w(L, t)$ along with a small rotation angle $\alpha=\arctan \left[w^{\prime}(L, t)\right]$. Thus the vertical displacement of magnet $\mathrm{D}$ can be evaluated by $w(L, t)+\frac{a \sin \alpha}{2}-D_{i}$, where $a$ is the side length of magnet $\mathrm{D}$ and $D_{i}$ represents the coordinate of magnet $i$ in y-direction $\left(D_{A}=\right.$ $\left.d_{g}, D_{B}=0, D_{c}=-d_{g}\right)$. Then the horizontal displacement of magnet $\mathrm{D}$ can be evaluated by $\Delta x=\frac{a}{2}(1-\cos \alpha)$. Since $\alpha \approx 0, \Delta x \approx 0$. So the vector $\boldsymbol{r}_{\boldsymbol{i D}}$ becomes

$$
\boldsymbol{r}_{i D}=\left[\begin{array}{ll}
-d_{0} & w(L, t)-D_{i}
\end{array}\right]
$$

and

$$
\begin{aligned}
\boldsymbol{m}_{D} & =\left[\begin{array}{ll}
M_{D} V_{D} \cos \alpha & M_{D} V_{D} \sin \alpha
\end{array}\right] \\
\boldsymbol{m}_{i} & =\left[\begin{array}{ll}
-M_{i} V_{i} & 0
\end{array}\right]
\end{aligned}
$$

where $M_{i}$ and $V_{i}$ represent the magnetization intensity and the volume of magnet $i$ respectively. So the potential energy generated by magnet $i$ upon D can be obtained by

$$
U_{m i D}=\frac{\mu_{0} M_{D} V_{D} M_{i} V_{i}\left(-\left(w(L, t)-D_{i}\right)^{2}+2 d_{0}^{2}-3 d_{0}\left(w(L, t)-D_{i}\right) w^{\prime}(L, t)\right)}{4 \pi \sqrt{\left(\left(w^{\prime}(L, t)\right)^{2}+1\right)}\left(\left(w(L, t)-D_{i}\right)^{2}+d_{0}^{2}\right)^{5 / 2}}
$$


Using Galerkin's concept, the transverse deflection $w(x, t)$ can be approximated by the linear combination of modes of the beam as

$$
\begin{aligned}
& w(x, t)=\sum_{j=1}^{N} \phi_{j}(x) q_{j}(t) \\
& \phi_{j}(x)= \begin{cases}\phi_{j 1}(x), & 0 \leq x<l_{p} \\
\phi_{j 2}(x), & l_{p} \leq x \leq L\end{cases} \\
& \phi_{j 1}(x)=C_{1} \cos \left(\beta_{j 1} x\right)+C_{2} \sin \left(\beta_{j 1} x\right)+C_{3} \cosh \left(\beta_{j 1} x\right)+C_{4} \sinh \left(\beta_{j 1} x\right) \\
& \phi_{j 2}(x)=P_{1} \cos \left(\beta_{j 2} x\right)+P_{2} \sin \left(\beta_{j 2} x\right)+P_{3} \cosh \left(\beta_{j 2} x\right)+P_{4} \sinh \left(\beta_{j 2} x\right)
\end{aligned}
$$

where $\phi_{j 1}(x)$ and $\phi_{j 2}(x)$ represent the linear mode shapes of the beams with and without the piezoelectric layers, respectively. $C_{k}$ and $P_{k}(k=1,2,3,4)$ are the constant coefficients derived from boundary conditions, $\beta_{j 1}$ and $\beta_{j 2}$ are the eigenvalues of the characteristic equation.

The geometric, material, electromechanical and magnetic parameters are given in Table 1.

Table 1. Model parameters used for numerical and experimental studies.

\begin{tabular}{lll}
\hline Symbol & Parameter & Value \\
\hline Substrate properties & & \\
\hline$L$ & Length & $135 \mathrm{~mm}$ \\
$b_{\mathrm{s}}$ & Width & $8 \mathrm{~mm}$ \\
$h_{\mathrm{s}}$ & Thickness & $0.12 \mathrm{~mm}$ \\
$\rho_{\mathrm{s}}$ & Density & $7800 \mathrm{~kg} / \mathrm{m}^{3}$ \\
$E_{\mathrm{s}}$ & Young's modulus & $205 \mathrm{Gpa}$ \\
\hline Piezoelectric laminate properties & \\
\hline$L_{\mathrm{p}}$ & Length & $5 \mathrm{~mm}$ \\
$b_{\mathrm{p}}$ & Width & $5 \mathrm{~mm}$ \\
$h_{\mathrm{p}}$ & Thickness & $0.15 \mathrm{~mm}$ \\
$\rho_{\mathrm{p}}$ & Density & $1785 \mathrm{~kg} / \mathrm{m}^{3}$ \\
$E_{\mathrm{p}}$ & Young's modulus & $2 \mathrm{Gpa}$ \\
$e_{31}$ & Coupling coefficient & $23 \times 10^{-10} \mathrm{C} / \mathrm{N}$ \\
$\varepsilon_{33}{ }^{3}$ & Permittivity constant & $1.06 \times 10^{-10} \mathrm{~F} / \mathrm{m}$ \\
\hline Magnet properties & & \\
\hline$m$ & Mass & $5.6 \mathrm{~g}$ \\
$A$ & Area & $10 \times 20 \mathrm{~mm}$
\end{tabular}




\begin{tabular}{lll}
$a$ & Thickness & $5 \mathrm{~mm}$ \\
$B_{r}$ & Residual flux density of magnet & $1.48 \mathrm{~T}$ \\
$d_{g}$ & Gap distance between fixed magnets & $20 \mathrm{~mm}$ \\
\hline
\end{tabular}
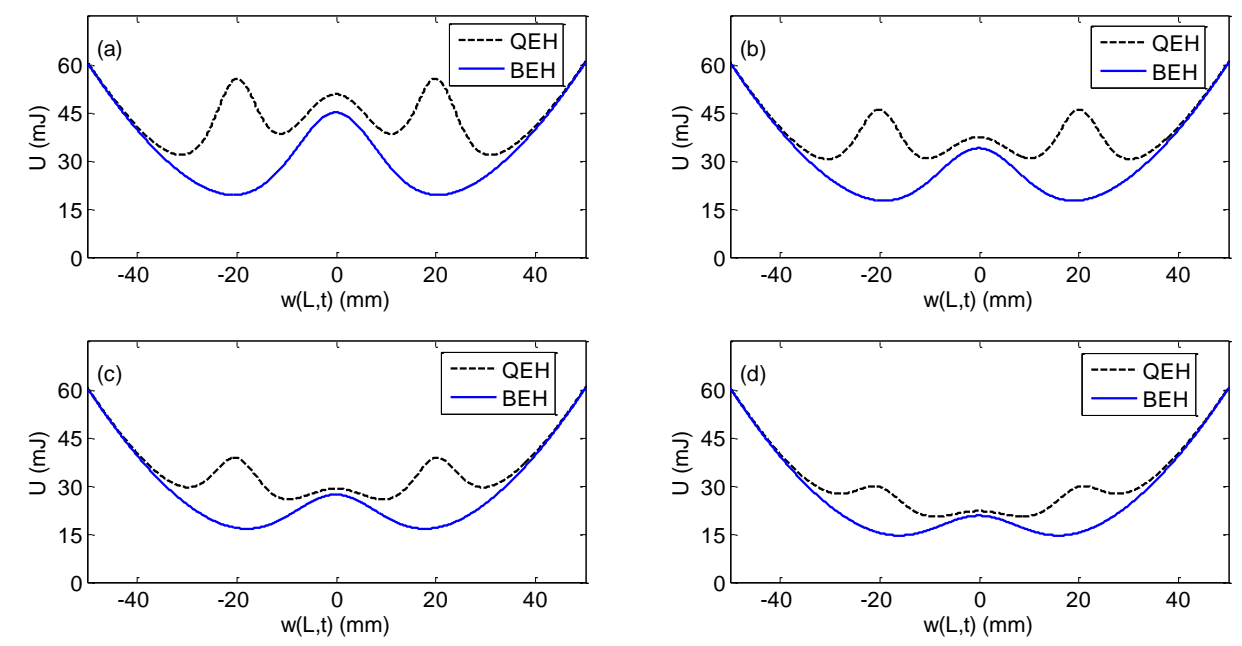

Fig. 4. Potential energy of QEH and BEH for different separation distances: (a) $d=3.5 \mathrm{~mm}$, (b) $d=5 \mathrm{~mm}$, (c) $d=7 \mathrm{~mm}$, (d) $d=9 \mathrm{~mm}$.

By Eq. (7), for magnets A, B and C, their magnetic potential energies can be calculated respectively. Then by Eq. (1), the whole magnetic potential energy of the QEH can be obtained. It should be noted that the total potential energies of both the QEH and the BEH consist of two parts: the beam's elastic potential energy and the magnetic one. Fig. 4 shows the functions of total potential energy of the QEH and the BEH for different separation distances $(d=3.5 \mathrm{~mm}, 5 \mathrm{~mm}, 7 \mathrm{~mm}$ and $9 \mathrm{~mm})$. It can be seen that the BEH has two potential wells and one barrier between them, while the QEH has four potential wells and three barriers between them. It is evident that the barriers of the QEH are lower than those of the BEH for the identical separation distance. This implies that the QEH needs a relatively lower excitation energy to cross the potential barrier than the $\mathrm{BEH}$.

\section{Validation experiments and discussions}

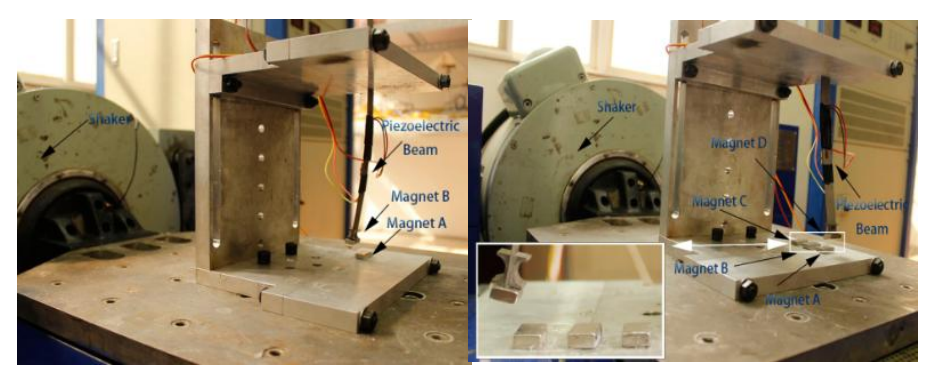

(a) BEH

(b) QEH

Fig. 5. Prototype of the energy harvester: (a) BEH, (b) QEH.

To validate the advantages of the $\mathrm{QEH}$, corresponding experiments are carried out. The experimental setups are as shown in Fig. 5. Figs. 5(a) and 5(b) show the BEH 
and QEH, respectively. The QEH consists of a bimorph piezoelectric beam with a tip magnet, a fixture and three fixed magnets. For the piezoelectric beam, its substrate layer is made of stainless steel with dimension $130 \times 8 \times 0.13 \mathrm{~mm}^{3}$. Two piezoelectric patches with dimension $25 \times 5 \times 0.15 \mathrm{~mm}^{3}$ are bonded on the substrate. As to magnets, four neodymium magnets have the identical dimension $20 \times 10 \times 5 \mathrm{~mm}^{3}$. Two edge distances between the three fixed magnets both are $10 \mathrm{~mm}$. For comparison, the same separation distance $(d=5 \mathrm{~mm})$ is set in experiment for the QEH and BEH. The accelerometer was mounted on the top of shaker (DONGLING ES-1-150) to monitor its acceleration. An oscilloscope (DH5922) is used to record the output voltage and strain of the energy harvester. The base accelerations are chosen as $a_{b}=0.7 \mathrm{~g}$ and 1.0 $\mathrm{g}$ ( $a_{b}$ is the peak-zero amplitude, and $g$ is the gravity acceleration). First the sweeping-frequency experiments are performed. We increase (or decrease) the excitation frequency at a fixed rate of $0.1 \mathrm{~Hz} / \mathrm{s}$. The frequency range is set from $5 \mathrm{~Hz}$ to $20 \mathrm{~Hz}$, and the exciting amplitudes are chosen as $a_{b}=0.7 \mathrm{~g}$ and $a_{b}=1.0 \mathrm{~g}$.
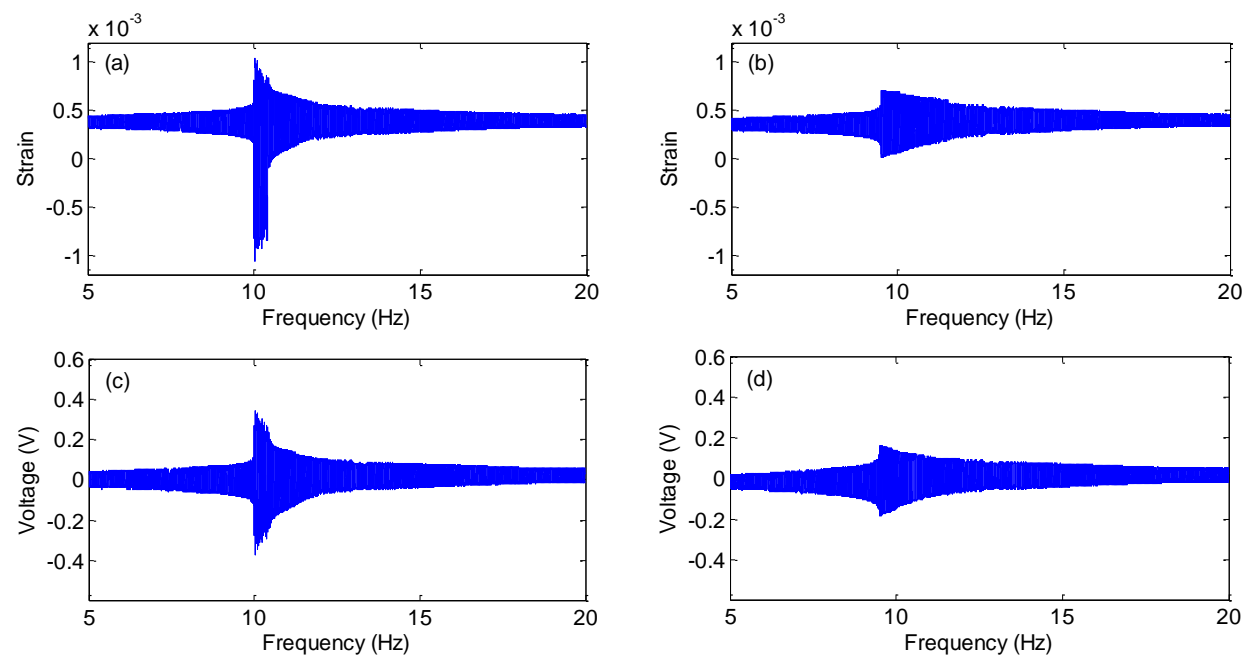

Fig. 6. Strains and voltages of BEH for sweeping frequency $\left(a_{b}=0.7 \mathrm{~g}\right)$ : (a, c) forward sweeping, (b, d) reverse sweeping.
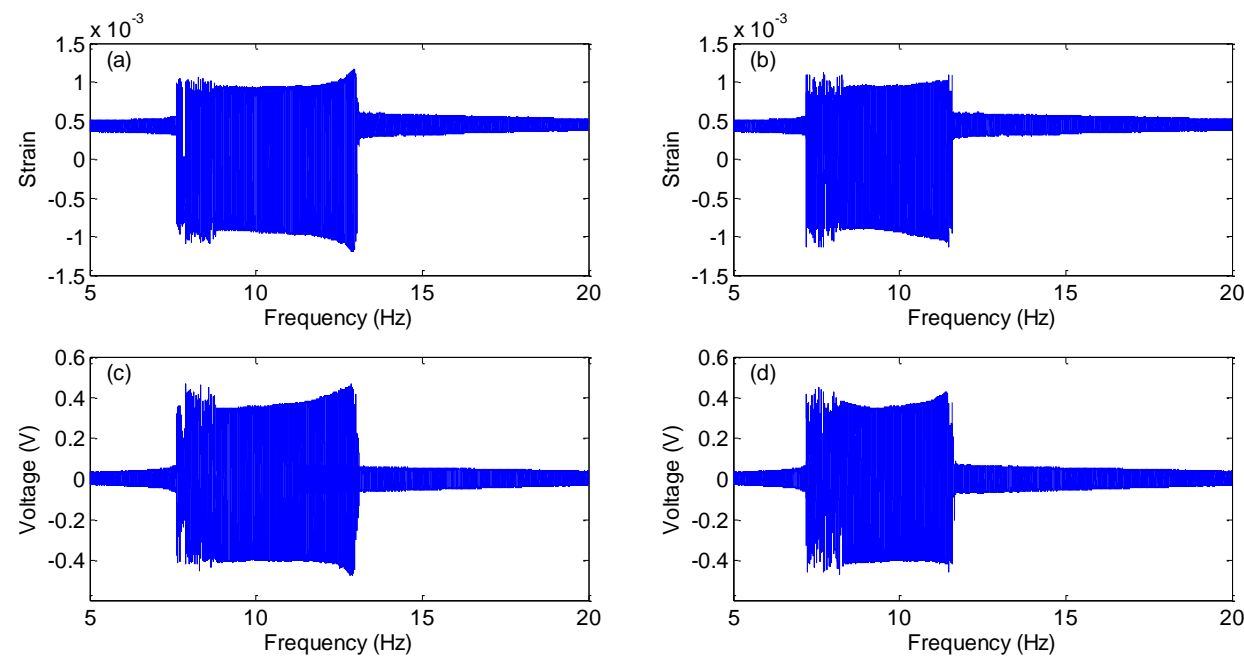

Fig. 7. Strains and voltages of QEH for sweeping frequency $\left(a_{b}=0.7 \mathrm{~g}\right)$ : $(\mathrm{a}, \mathrm{c})$ forward sweeping, (b, d) reverse sweeping.

Figs. 6 and 7 illustrate the strains and voltages of the QEH and $\mathrm{BEH}$ for 
frequency-sweeping excitation with $a_{b}=0.7 \mathrm{~g}$. This excitation level is relatively low for the BEH and can't cause snap-through between its potential wells. Hence the response of the BEH keeps intra-well oscillation, and the output voltage generated by it is small (Figs. 6(c) and 6(d)). In contrast, at this excitation level the QEH can easily pass through its potential barriers and leads to snap-through between the potential wells, which makes the strain increase sharply and produces a high voltage within several ranges of frequency (7.6-12.8 Hz for forward sweeping, 7.2-11.6 Hz for reverse sweeping) (Figs. 7(c) and 7(d)). Therefore, we could conclude that the QEH can go to snap-through easier under the low excitation and thus harvest more excitation energy than the $\mathrm{BEH}$.
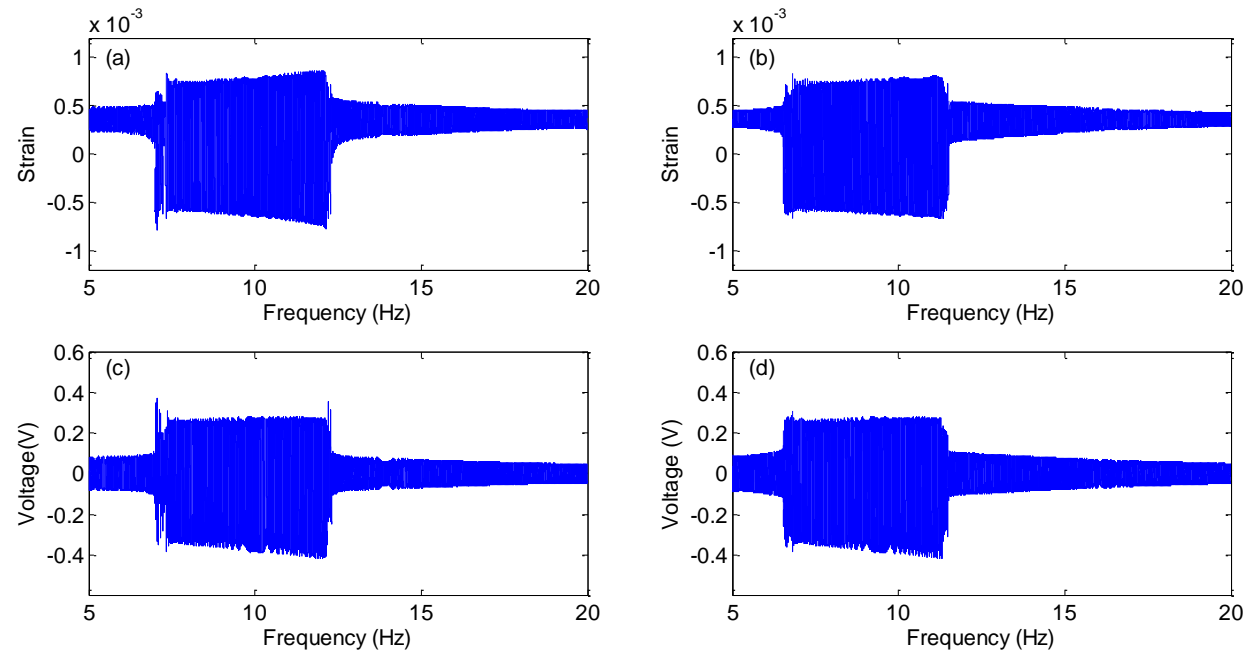

Fig. 8. Strains and voltages of BEH for sweeping frequency $\left(a_{b}=1.0 \mathrm{~g}\right)$ : $(\mathrm{a}, \mathrm{c})$ forward sweeping, (b, d) reverse sweeping.
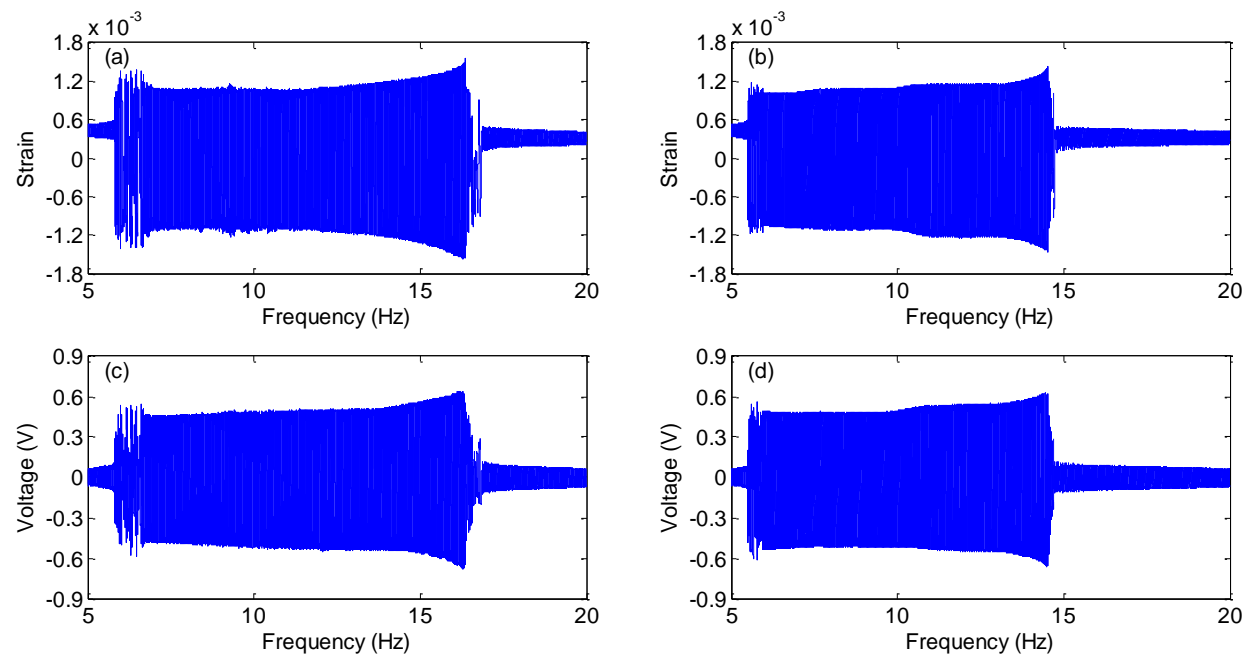

Fig. 9. Strains and voltages of QEH for sweeping frequency at $a_{b}=1.0 \mathrm{~g}$ : (a, c) forward sweeping, (b, d) reverse sweeping.

Next as the excitation increases to $a_{b}=1.0 \mathrm{~g}$, there will appear the phenomenon of snap-through between the potential wells in the BEH as well, but the difference is obvious. Figs. 8 and 9 show the strains and output voltages of the BEH and QEH under the frequency sweeping with $a_{b}=1.0 \mathrm{~g}$. It can be seen that the QEH can elicit snap-through within nearly the whole range of 5.8-16.6 Hz in forward sweeping and 
5.3-14.7 Hz in reverse sweeping (Figs. 9(a) and 9(b)), whereas the BEH only have snap-through within 7.1-12.3 Hz (forward sweeping) and 6.8-11.4 Hz (reverse sweeping) (Figs. 8(a) and 8(b)). As to output voltages, the QEH gives out a much higher voltage in a much wider bandwidth than the $\mathrm{BEH}$. The highest voltage generated by the $\mathrm{QEH}$ can reach $0.62 \mathrm{~V}$, whereas that generated by the $\mathrm{BEH}$ is only $0.41 \mathrm{~V}$. Hence the QEH can harvest the excitation energy more effectively than the $\mathrm{BEH}$.
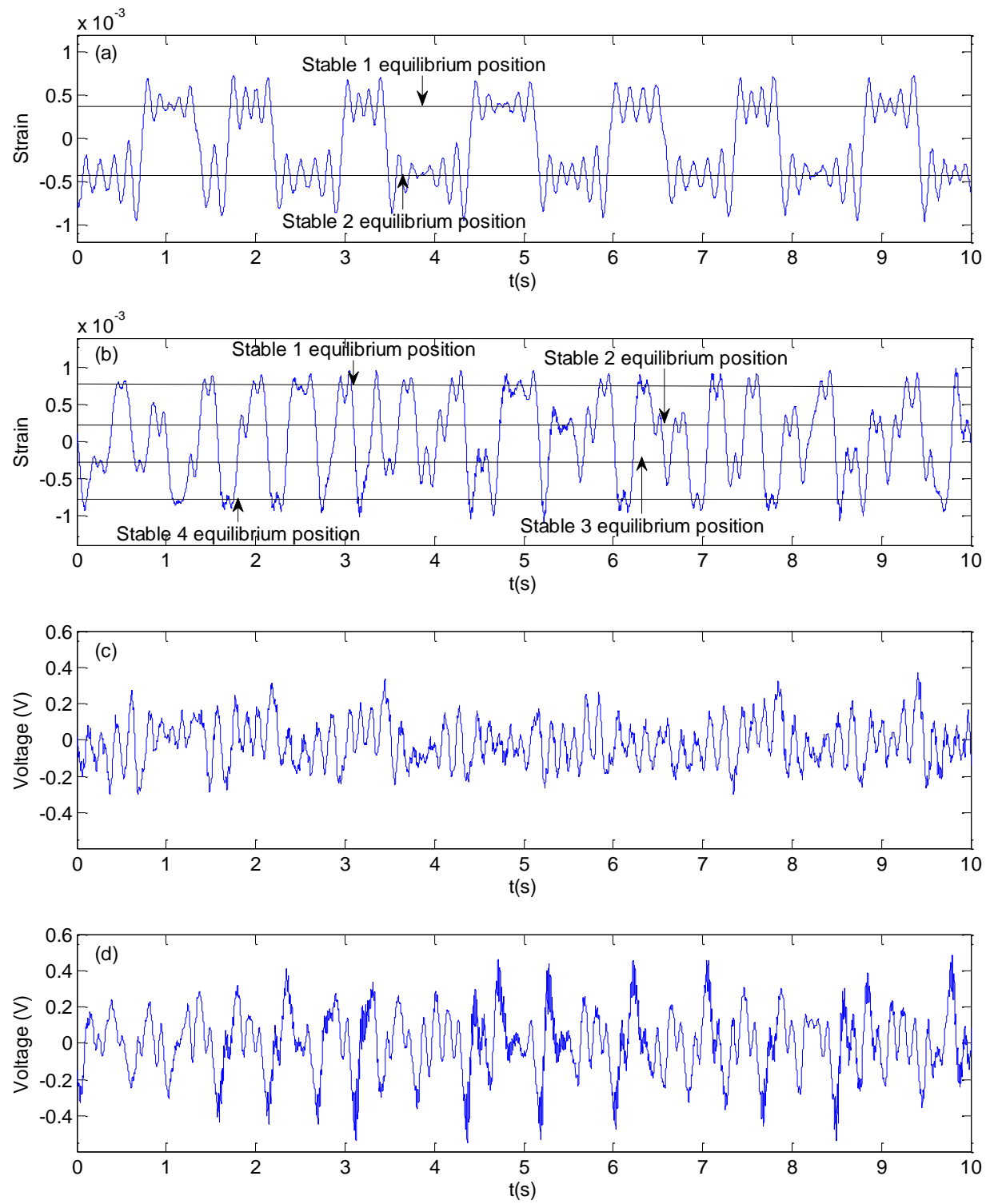

Fig. 10. Strain and voltage responses of snap-through: $(a, c) B E H,(b, d) Q E H$.

In order to further study the snap-through phenomena in the $\mathrm{QEH}$ and $\mathrm{BEH}$, the excitation in experiment is fixed as $f=7 \mathrm{~Hz}$ and $a_{b}=1.0 \mathrm{~g}$. The corresponding results are shown in Fig. 10. From Fig. 10(a), it is shown that the BEH has two stable equilibrium positions and its response jumps between them in the state of snap-through. However, its response generally oscillates around an equilibrium position for a while before jumping to another, i.e., there exists a time interval between two jumps. Then for the QEH, there exist four stable equilibrium positions. 
From Fig. 10(b) it is evident that the response can jump between the first and the second positions, the first and the third ones, the second and the third ones, even the first and the fourth ones. Due to the existence of multi-stable positions and the low barriers, the jump happens more easily and can be repeated in the QEH. So there appears a dense jump in its response. The snap-through of the QEH between the nonadjacent stable positions brings about a large amplitude, which can give out a higher output voltage than that of the BEH (Figs. 10(c) and 10(d)).
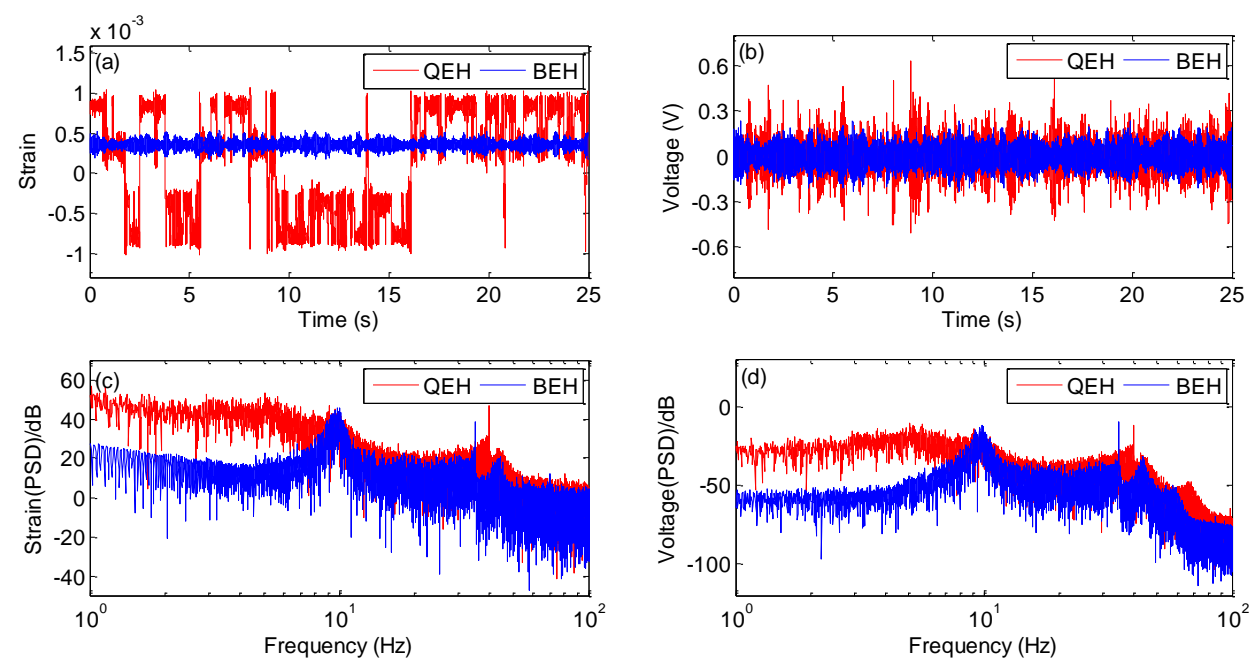

Fig. 11. (a) Strain, (b) voltage, (c) strain PSD, (d) voltage PSD at $D=0.03 \mathrm{~g}^{2} \mathrm{~Hz}^{-1}$ for QEH and BEH.

To compare their abilities of harvesting the random energy in environment, the base excitation is designated as white Gaussian noise for the $\mathrm{BEH}$ and QEH, whose power spectral density $(D)$ is designed to vary from $D=0.01$ to $D=0.1 \mathrm{~g}^{2} \mathrm{~Hz}^{-1}$. The frequency bandwidth of the base excitation is set as $\Delta f=5-200 \mathrm{~Hz}$. The experimental results are shown in Figs. 11-13, in terms of strains, output voltages, spectra of strain, spectra of output voltages and RMS voltages. Figs. 11 and 12 illustrate the responses and voltages of the two harvesters at the two levels of excitation respectively. When the intensity is relatively low $\left(D=0.03 \mathrm{~g}^{2} \mathrm{~Hz}^{-1}\right)$, in contrast to the intrawell oscillation of the BEH, the QEH can undergo jumping(snap-through) between the four potential wells (Fig. 11(a)), which bring about a larger output voltage (Fig. 11(b)). It should be noted that in the QEH's time history of voltage there are some sharp peaks, which corresponds to the jumps between the potential wells in strain responses. As for spectrum, Figs. 10(c) and 10(d) give the PSDs of strain responses and output voltages for the $\mathrm{BEH}$ and $\mathrm{QEH}$, which prove the advantage of the QEH.
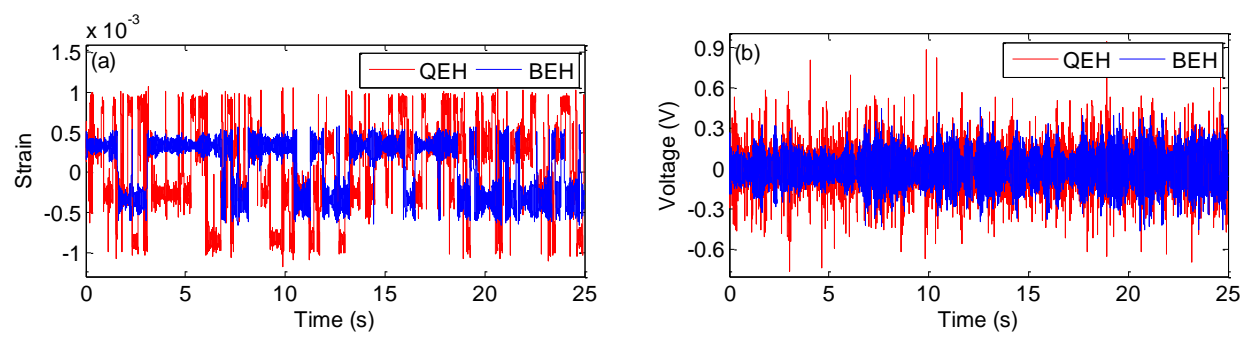

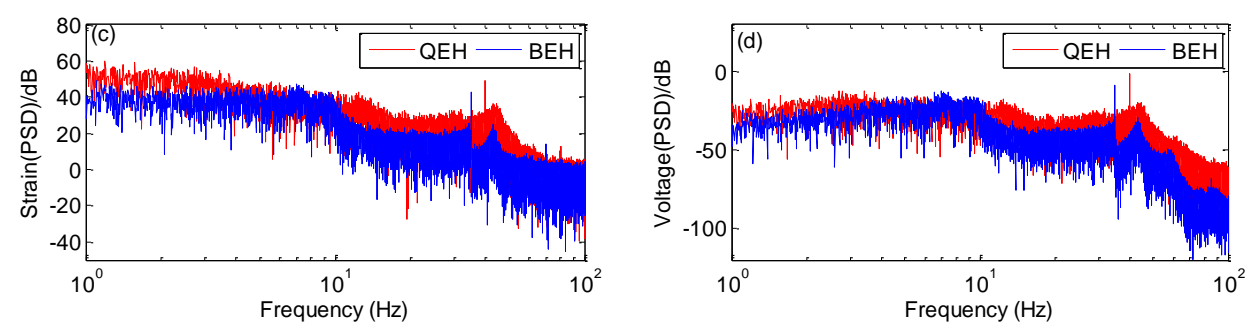

Fig. 12. (a) Strain, (b) voltage, (c) strain PSD, (d) voltage PSD for $D=0.05 \mathrm{~g}^{2} \mathrm{~Hz}^{-1}$ for QEH and BEH.

Next the random excitation is increased to $D=0.05 \mathrm{~g}^{2} \mathrm{~Hz}^{-1}$, the results are shown in Fig. 12. At this intensity, the BEH starts interwell jumping as well, whereas the QEH has arrived at the state of frequently jumping. Thus the QEH can create a much larger output voltage (Fig. 12(b)). From the PSDs of the strains and voltages (Figs. 12(c) and 12(d)), the same trend can be found. Fig. 13 illustrates the RMS voltages of the $\mathrm{BEH}$ and $\mathrm{QEH}$ over the whole range of excitation intensity $D$. It is shown that the $\mathrm{QEH}$ can produce the higher voltage over nearly the whole range of $D$ than the BEH.

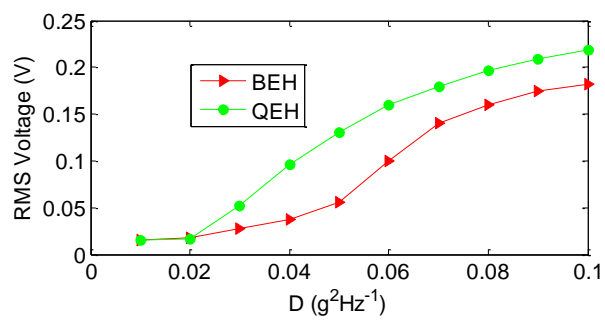

Fig. 13. RMS voltages obtained from $\mathrm{BEH}$ and $\mathrm{QEH}$.

\section{Conclusion}

In this paper, we developed a quad-stable piezoelectric device to harvest the base random vibration energy. By mounting and adjusting three magnets on the fixture, the magnetic forces can make the QEH realize four stable equilibrium positions. The theoretical analysis of potential energy shows that the proposed QEH possesses the lower and wider potential wells than the BEH. The experiments of frequency sweeping and random excitation are carried out, which indicated that the QEH had a wider bandwidth of snap-through and could easily realize snap-through between the potential wells at random excitations. The QEH can generate higher RMS output voltages and maximum voltages than the BEH. So the proposed QEH is preferable in harvesting environment energy.

\section{Acknowledgment}

The support of the National Science Foundation of China (Grant No. 11172235) is greatly acknowledged.

\section{References}

[1] B. Andò, S. Baglio, A.R. Bulsara, V. Marletta, A bistable buckled beam based approach for vibrational energy harvesting, Sens. Actuators A: Phys. 211 (2014) 153-161. 
[2] P. Glynne-Jones, M.J. Tudor, S.P. Beeby, N.M. White, An electromagnetic vibration-powered generator for intelligent sensor systems, Sens. Actuators A: Phys.110 (2004) 344-349.

[3] E. Lefeuvre, A. Badel, C. Richard, L. Petit, D. Guyomar, A comparison between several vibration-powered piezoelectric generators for standalone systems, Sens. Actuators A: Phys. 126 (2006) 405-416.

[4] M. Ferrari, V. Ferrari, M. Guizzetti, B. Andò, S. Baglio, C. Trigona, Improved energy harvesting from wideband vibrations by nonlinear piezoelectric converters, Sensors Actuat. A Phys. 162 (2010) 425-431.

[5] G. Gatti, M.J. Brennan, M.G. Tehrani, D.J. Thompson, Harvesting energy from the vibration of a passing train using a single-degree-of-freedom oscillator, Mech. Syst. Signal Process.66-67 (2016) 785-792.

[6] A. Camarda, A. Romani, E. Macrelli, M. Tartagni, A $32 \mathrm{mV} / 69 \mathrm{mV}$ input voltage booster based on a piezoelectric transformer for energy harvesting applications, Sens. Actuators A: Phys. 232 (2015) 341-352.

[7] J. Xu, J. Tang, Linear stiffness compensation using magnetic effect to improve electro-mechanical coupling for piezoelectric energy harvesting, Sens. Actuators A: Phys. 235 (2015) 80-94.

[8] A. Erturk, Piezoelectric energy harvesting for civil infrastructure system applications: Moving loads and surface strain fluctuations, J. Intell. Mater. Syst. Struct.22 (2011) 1959-1973.

[9] E. Felix, G. Wang, B. Cain, Experimental investigation of galloping piezoelectric energy harvesters with square bluff bodies, Smart Mat. Struct. 23 (2014) 104012.

[10] L. Zuo, X.D. Tang, Large-scale vibration energy harvesting, J. Intel. Mat. Sys. Struct. 24 (2013) 1405-1430.

[11] H. Vocca, I. Neri, F. Travasso, L. Gammaitoni, Kinetic energy harvesting with bistable oscillators, Appl. Energy 97 (2012) 771-776.

[12] R.L. Harne, K.W. Wang, A review of the recent research on vibration energy harvesting via bistable systems, Smart Mater. Struct. 22 (2013) 023001.

[13] S.P. Pellegrini, N. Tolou, M. Schenk, J.L. Herder, Bistable vibration energy harvesters: a review, J. Intell Mater. Syst. Struct. 24 (2013) 1303-1312.

[14] V.R. Challa, M.G. Prasad, Y. Shi, F.T. Fisher, A vibration energy harvesting device with bidirectional resonance frequency tenability, Smart Mater. Struct.17 (2008) 015035.

[15] D. Zhu, S. Roberts, J. Tudor, S. Beeby, Closed loop frequency tuning of a vibration-based micro-generator, Proc. Power MEMS, Sendai Japan, 2008, pp. 229-232 (9-12 November).

[16] S. Aline, P. De, J.I. Daniel, A.S. Marcelo, Energy harvesting in a nonlinear piezomagnetoelastic beam subjected to random excitation, Mech. Syst. Signal Process.54-55 (2015) 405-416.

[17] A. Erturk, J. Hoffmann, D.J. Inman, A piezomagnetoelastic structure for broadband vibration energy harvesting, Appl. Phys. Lett. 94 (2009) 254102.

[18] A. Erturk, D.J. Inman, Broadband piezoelectric power generation on high-energy orbits of the bistable Duffing oscillator with electromechanical coupling, J. Sound Vib.330 (2011) 2339-2353.

[19] L. Tang, Y. Yang, C.K. Soh, Improving functionality of vibration energy harvesters using 
magnets, J. Intell. Mater. Syst. Struct. 23 (2012) 1433-1449.

[20] J.T. Lin, B. Lee, B. Alphenaar, The magnetic coupling of a piezoelectric cantilever for enhanced energy harvesting efficiency, Smart Mater. Struct. 19 (2010) 045012.

[21] S. Zhou, J. Cao, A. Erturk, J. Lin, Enhanced broadband piezoelectric energy harvesting using rotatable magnets, Appl. Phys. Lett. 102 (2013) 173901.

[22] L. Tang, Y. Yang, A nonlinear piezoelectric energy harvester with magnetic oscillator, Appl. Phys. Lett. 101 (2012) 094102.

[23] Y.J. Gao, Y.G. Leng, S.B. Fan, Z.H. Lai, Performance of bistable piezoelectric cantilever vibration energy harvesters with an elastic support external magnet, Smart Mater. Struct. 23 (2014) 095003.

[24] J.Y. Jung, P. Kim, J. Lee, J.W. Seok, Nonlinear dynamic and energetic characteristics of piezoelectric energy harvester with two rotatable external magnets, Int. J. Mech. Sci. 92 (2015) 206-222.

[25] P. Kim, J. Seok, A multi-stable energy harvester: dynamic modeling and bifurcation analysis, J. Sound Vib. 333 (2014) 5525-5547.

[26] S.C. Stanton, C.C. McGehee, B.P. Mann, Nonlinear dynamics for broadband energy harvesting: investigation of a bistable piezoelectric inertial generator, Physica D 239 (2010) 640-653.

[27] K. Yung, P. Landecker, D. Villani, An analytic solution for the force between two magnetic dipoles, Magn. Electric. Separat. 9 (1998) 39-52.

[28] H. Wu, L. Tang, C. K. Soh, Development of a broadband nonlinear two-degree-of-freedom piezoelectric energy harvester, J. Intell. Mater. Syst. Struct. 25 (2014) 1875-1889. 


\section{Biographies}

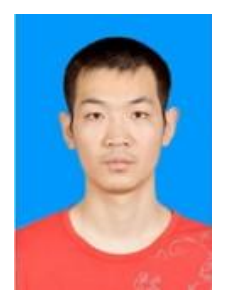

Zhi-yong Zhou received his B.E. and M.S. degrees from the Henan University of China in 2009 and 2012, respectively. He is currently a Ph.D. student in Department of Engineering Mechanics, Northwestern Polytechnical University, China. His research interests include piezoelectric vibration energy harvesting, nonlinear dynamics and mechanical vibration.

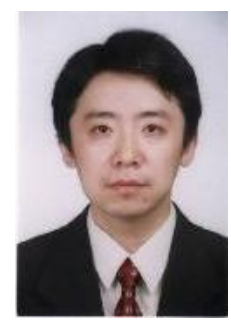

Wei-yang Qin is Professor of Northwestern Polytechnical University, China. He received the B.S. degrees in Applied Mechanics from Fudan University, China, in 1989. He received the M.S. and Ph.D. degrees from Northwestern Polytechnical University, China, in 1992 and 2001, respectively. He has been a director of the professional committee of modal and vibration testing of Vibration Engineering Society of China. Dr. Wei-yang Qin's research interests are nonlinear dynamics, mechanical vibration, signal processing, piezoelectric vibration energy harvesting and rotor dynamics.

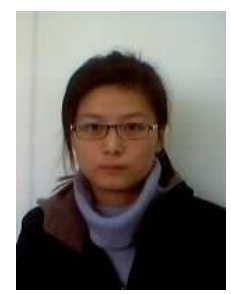

Pei Zhu received her M.S. degree from the Henan University of China in 2013. She is currently working toward the Ph.D. degree in Department of Engineering Mechanics, Northwestern Polytechnical University, China. Her research interests include piezoelectric vibration energy harvesting, nonlinear dynamics and rotor dynamics. 